\title{
A STOCHASTIC FLOW-DEPENDENT MODEL FOR PATH FLOW ESTIMATION
}

\author{
経路交通量推定のための確率的フローディペンデントモデル
}

\author{
Lin Cheng $^{*}$ Yasunori Iida $^{* *}$ Nobuhiro Uno ${ }^{* *}$
}

\section{Introduction}

With the rapid development of vehicle information and communication technology, it is urgently required to collect up-to-date information about the state of road network, such as link flows, locations of congestion, path flows and path travel times. For example, dynamic path guidance requires estimates of path travel times, while traffic detectors are set up only at some sections of road network to collect such data as link flows. These observations need to be complemented by some processors, in order to build a complete picture of current network states.

Iida and Asakura (1988) suggested a bi-level programming model consisting of systematically objective optimization and deterministic assignment, to calculate network information such as link flows, OD matrix. Yang et al. (1992) investigated alternative solutions and indicated that the upper level problem can allow approximately for user responses once the sensitivities of link flows to trip table in the lower level are passed back. Although OD demand and link flow can be determined in the bilevel model, the method, as it is known, does not in general uniquely define the path flows.

Sherali et al. (1994) proposed a linear programming Path Flow Estimator (abbreviated to PFE) for estimating user equilibrium path flows, which may be aggregated to yield an OD matrix. In general, the PFE can be regarded as a practical tool to estimate path flows and OD flows. The PFE requires only some observations on link flows. Bell and Iida (1997) proposed a non-linear programming PFE to estimate stochastic user equilibrium path flows, which may be used for estimating travel time reliability.

The purpose of this study is to develop the path flow estimator that can produce estimates of path flow, the correspondent cost and the OD trip table, from observations of link traffic volumes. This study makes

Keyword: path flow estimation, congested network,

overload delay, observed link flow

*student member of JSCE, Department of Civil Engineering,

School of Engineering, Kyoto University

Sakyoku, Kyoto, Japan, T: 075-753-5126, F: 075-7535907

chengrourbanfac.kuciv. kyouto-u.ac.jp

** Fellow of JSCE, Ph.D., Professor,

Department of Civil Engineering, Kyoto University

***Regular Member of JSCE, Ph.D., Associate Professor,

Department of Civil Engineering, Kyoto University two improvements on the stochastic PFE proposed by Bell and Iida (1997). First, the link travel time is assumed to be a monotone increasing function of the link flow, or say, the running time is a flow-dependent function. Second, the improved PFE explicitly considers overload delay on the road network.

The paper is organized as follows. The basic idea of the flow-dependent PFE is described in Chapter 2. Chapter 3 presents the iterative balancing method to solve our flow-dependent PFE. Chapter 4 demonstrates two numerical examples. Chapter 5 briefly accounts for the detector allocation problem for the PFE, by the sensitivity analysis of the stochastic equilibrium model. Some conclusions are given in Chapter 6.

\section{Flow-dependent PFE Model}

There are perhaps two aspects of awareness about congestion effects on the road network. The one is that the congestion may be described by using the increasing travel time function against the link flow; the other is the overload delay occurred at the link exit (Inoue, 1986). Both of two congestion effects are considered in this paper that distinguishes from the pioneering work (Bell and Iida, 1997), in which the travel time of each link is treated as constant, unrelated to the volume of the link flow. The flow-dependent PFE assumes that the travel cost is comprised of the running time and the delay. The running time is estimated by the flow-dependent travel time function, and the delay is associated with the Lagrange multiplier of the capacity constraint. The framework of the flow-dependent PFE is illustrated in Table 1. In this chapter some properties of traffic flow and representation of link travel cost are firstly discussed. Then the nature of logit choice model is focused. After that the flow-dependent PFE is formulated as a programming issue. The determination of parameter is described lastly, which is also included in the part of the stochastic assignment.

\subsection{Representation of Link Flow And Link Cost}

This study considers the traffic congestion under equilibrium state based on the previous research works (Inoue, 1986). To begin with, the traffic flow is approximately assumed to be a steady state. Fig. 1 shows an arbitrary link with flow $x_{a}$. The link traffic condition is assumed to partition into non-congested regime and congested regime, the length of which is $l_{l}$ 
Table 1. The Structure of Path Flow Estimator

\begin{tabular}{|c|c|c|c|}
\hline \multirow{4}{*}{$\begin{array}{l}\text { outer } \\
\text { loop }\end{array}$} & \multicolumn{3}{|c|}{ column generation } \\
\hline & \multirow{3}{*}{$\begin{array}{l}\text { middle } \\
\text { loop }\end{array}$} & balancing & nk cost and flow following performance functions \\
\hline & & \multirow{2}{*}{$\begin{array}{l}\text { inner } \\
\text { loop }\end{array}$} & balancing network flows and observed link flows by logit-model \\
\hline & & & delaying occurring on active links \\
\hline
\end{tabular}

and $l_{2}$ respectively. Under the equilibrium flow of steady state, the length of congested regime becomes steady.

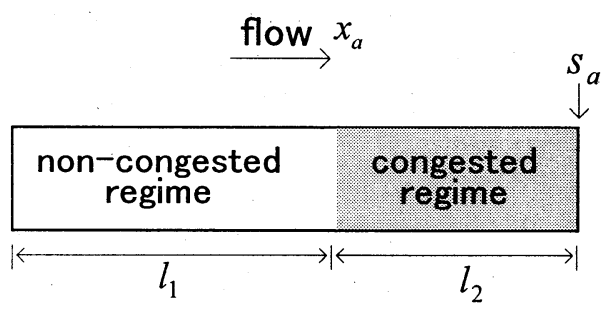

Fig. 1 The link flow of congestion

Let $s_{a, \max }$ represents the roadway capacity of the link $a$, and $s_{a}$ represents the exit capacity of the link $a$. In general, the link exit capacity $s_{a}$ is less than the road capacity $s_{a, \max }$, because two major traffic streams cross each other at the intersection, and the safe and efficient operation of the intersection requires some kind of control measure like signal control. The signalized or non-signalized intersection crossed by links allows the link flow $x_{a}$ to pass through, which must be not more than the corresponding exit capacity $s_{a}$. If the flow temporarily exceeds the link exit capacity, the congestion generates from the exit end of the link.

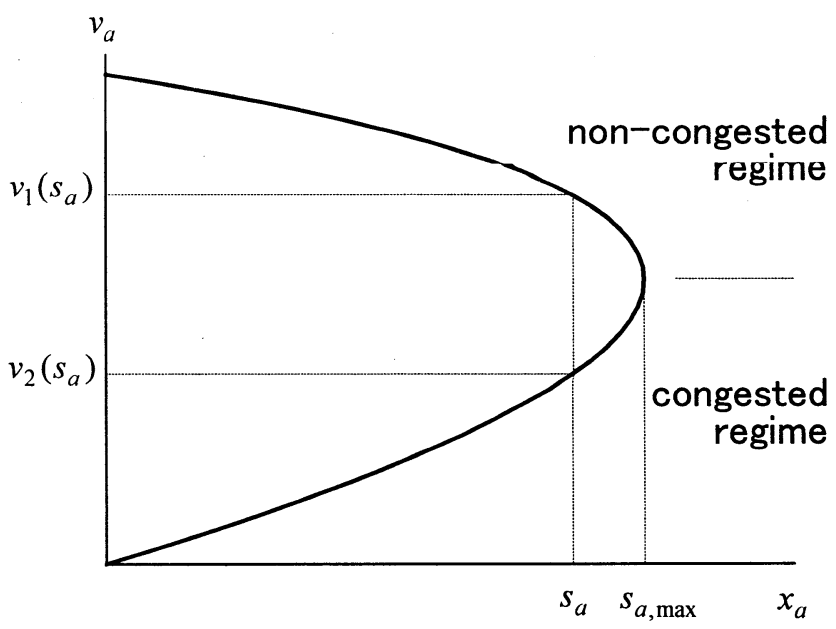

Fig. 2 The relationship of speed and flow

As illustrated in Fig. 2, the speed-flow relationship of the congested link is represented by $v_{l}\left(x_{a}\right)$ in noncongested regime and $v_{2}\left(x_{a}\right)$ in non-congested regime. The function $v_{l}\left(x_{a}\right)$ is monotone decreasing, while $v_{2}\left(x_{a}\right)$ is monotone increasing. In the equilibrium state, the travel time $t_{a}$ on the link $a$ is given by

$$
\begin{aligned}
t_{a} & =\frac{l_{1}}{v_{1}\left(s_{a}\right)}+\frac{l_{2}}{v_{2}\left(s_{a}\right)} \\
& =\frac{l_{1}+l_{2}}{v_{1}\left(s_{a}\right)}+\left[\frac{l_{2}}{v_{2}\left(s_{a}\right)}-\frac{l_{2}}{v_{1}\left(s_{a}\right)}\right]
\end{aligned}
$$

The first term of equation (1) represents the ordinary travel time, which can be estimated by some sort of travel time function such as BPR function. The second term corresponds to the waiting time or overload delay due to the congestion at the link exit, caused by deficiency of the link exit capacity. Therefore, the generalized link travel cost is comprised of the flowdependent running time and the waiting time. Fig. 3 shows the generalized travel time performance function used in this study.

If the flow is less than the link exit capacity, the flow can smoothly pass through the link, then no overload delay exists. Once if the flow reaches the link exit capacity, the congested regime appears in the link and the corresponding link flow becomes equal to the link exit capacity. This can be summarized as

$$
\left.\begin{array}{ll}
d_{a}=0 & \text { if } x_{a}<s_{a} \\
d_{a}>0 & \text { if } x_{a}=s_{a}
\end{array}\right\}
$$

The overload delay $d_{a}$ may be regarded as a penalty item added in the link travel time if the flow excesses the link exit capacity.

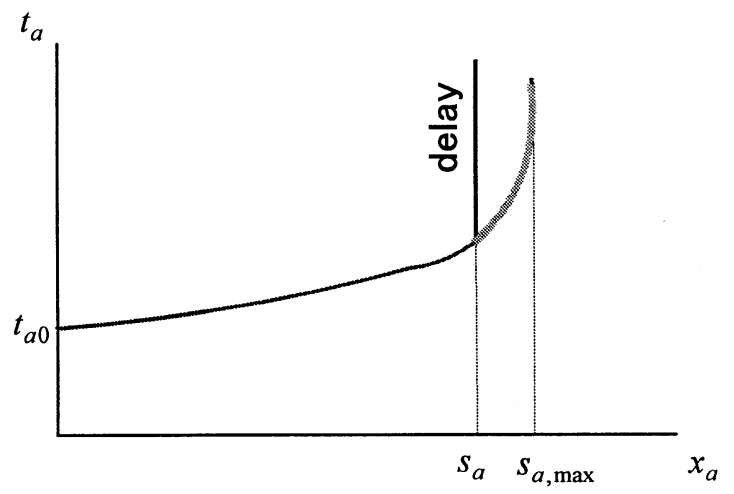

Fig. 3 Link travel time performance

\subsection{Nature of Logit Path Choice}

Consider a network composed of two paths $k$ and $k$ ' connecting one origin and one destination. The path choice probability is given by the well-known logit model. 


$$
\operatorname{Pr}[k]=\frac{\exp \left(-\theta C_{k}\right)}{\exp \left(-\theta C_{k}\right)+\exp \left(-\theta C_{k^{\prime}}\right)}
$$

where $C_{k}$ is the travel time on path $k, \theta$ is the dispersion parameter. Since the path flow $h_{k}$ is proportional to the path choice probability, it leads the following equivalent logit model with respect to path flows.

$$
\begin{aligned}
& h_{k} / h_{k^{\prime}}=\exp \left(-\theta C_{k}\right) / \exp \left(-\theta C_{k^{\prime}}\right) \\
& \text { or } \ln \left(h_{k} / h_{k^{\prime}}\right)=-\theta\left(C_{k}-C_{k^{\prime}}\right)
\end{aligned}
$$

This equation creates the relationship between of flow and cost in the stochastic PFE. Specifically speaking, the stochastic user equilibrium (SUE) is achieved when and only when the trip allocation between alternative paths satisfies the following alternative form of equation (5).

$$
\frac{1}{\theta} \ln h_{k}+C_{k}\left(h_{k}\right)=\frac{1}{\theta} \ln h_{k^{\prime}}+C_{k^{\prime}}\left(h_{k^{\prime}}\right)=u^{w}
$$

The equation (6) expresses the significant feature that each OD-pair $w$ holds a constant value $u^{w}$ respectively at the equilibrium optimum, which is called equilibrium cost of OD pair $w$. For the path $k$ within pair $w$, the constant value consists of the inherent travel cost $C_{k}\left(h_{k}\right)$ and the additional cost, $(1 / \theta) \ln h_{k}$, produced by correspondent path flows. It signifies that the logit model assigns the more OD flow to the lower cost path, the less OD flow to the higher cost path. It evidently differs from the shortest path assignment in which the OD flow gathers in the path of minimum cost. The logit choice model can be equivalently formulated as a mathematical programming problem, called stochastic user equilibrium, which can be found in Bell et al. (1997).

\subsection{Path Flow Estimator}

Because of uncertainty of path choice, and imperfect information about network state, an assumption included in UE is evidently violated and hence the stochastic user equilibrium is preferred to simulate drivers' path choice behaviours. The stochastic Path Flow Estimator is formulated as the following mathematical programming:

$\min f(\mathbf{h})=\frac{1}{\theta} \mathbf{h}^{\mathrm{T}}(\ln \mathbf{h}-1)+\sum_{a} \int_{0}^{x_{a}(\mathbf{h})} c_{a}(x) d x$

subject to

$$
\begin{gathered}
\mathbf{A}_{0} \mathbf{h} \leq \mathbf{s} \\
\mathbf{v}=\mathbf{A}_{\mathbf{1}} \mathbf{h}
\end{gathered}
$$

where $\mathbf{h}$ denotes the column vector of path flows. Matrix $\left[\mathbf{A}_{0}, \mathbf{A}_{1}\right]^{\mathrm{T}}$ represents the incidence relationship between the unobserved, observed link flows and path flows $\mathbf{h}$. Vector $\mathbf{v}$ is the observed link flow. $\mu_{0}, \mu_{1}$ are the Lagrange multiplier vector of capacity constraints and observation equations, respectively. In this study, it is assumed that the links are classified into the observed links and the unobserved ones. The observed link is equipped with the traffic detector. Conversely, no traffic detector is installed into the unobserved link.

The mathematical programming expressed by (7) can give the estimates of the path flows and OD flows by using the observed link flows. The mathematical programming includes two kinds of constraints: the link capacity constraint expressed by (7b) and the observed link flow constraint expressed by (7c). The traffic conditions on the unobserved links must be satisfied with the link capacity constraints. The constraint (7c) means that the estimated link flows must coincide with the observed link flows.

The Lagrange function of the PFE represented by (7) can be formed:

$$
L(\mathbf{h}, \boldsymbol{\mu}, \boldsymbol{\lambda})=f(\mathbf{h})+\boldsymbol{\mu}_{0}^{\mathrm{T}}\left(\mathbf{A}_{0} \mathbf{h}-\mathbf{s}\right)+\boldsymbol{\mu}_{1}^{\mathrm{T}}\left(\mathbf{v}-\mathbf{A}_{1} \mathbf{h}\right)
$$

At optimum, the first-order condition is

$$
\begin{gathered}
\frac{1}{\theta} \ln \mathbf{h}+\mathbf{C}+\mathbf{A}_{0}^{\mathrm{T}} \boldsymbol{\mu}_{0}=\mathbf{A}_{1}^{\mathrm{T}} \boldsymbol{\mu}_{1} \\
\frac{1}{\theta} \ln h_{k}+C_{k}+\sum_{a} \mu_{0 a} \delta_{a k}=\sum_{a} \mu_{1 a} \delta_{a k}
\end{gathered}
$$

where $\sum \mu_{0 a} \delta_{a k}$ denotes the sum of Lagrange multipliers with respect to the capacity-constraints of unobserved links on path $k$, and $\sum \mu_{1 a} \delta_{a k}$ is that of Lagrange multipliers with respect to observed links along path $k$, $C_{k}$ is the running time along path $k$, the element of travel time vector C. $C_{k}$ can be obtained by summing up the estimated running times of the links included in the path $k$.

Comparison between equation (9) and (6) might help us understand the difference between the ordinary SUE and the stochastic PFE in which the congestion is explicitly considered. There are some differences in the expression and interpretation of travel cost between the two equations. Firstly in the left-hand side of equation (9), the path travel cost is composed of the path running time $C_{k}$ and the overload delay expressed by $\sum \mu_{0 a} \delta_{a k}$. The Lagrange multiplier of the link capacity constraint is determined by the corresponding complementary slackness conditions:

$$
\left.\begin{array}{ll}
\mu_{a}=0 & \text { if } \quad s_{a}>\sum \delta_{a k} h_{k} \\
\mu_{a}>0 & \text { if } \quad s_{a}=\sum \delta_{a k} h_{k}
\end{array}\right\}
$$

The comparison between equation (2) and (10) shows both conditions are the same if let $d_{a}=\mu_{a}, x_{a}=\sum \delta_{a k} h_{k}$.

Secondly, the comparison of the right-hand side between equation (6) and (9) implies that the path flow pattern obtained by the stochastic PFE might be different from that obtained by the logit-type assignment because of the influence of the observed link flow constraint expressed by (7c). Judging from the optimality condition expressed by (6), the equilibrium cost of must be determined uniquely for each OD pair in the logit-type assignment. However, $\sum \mu_{1 a} \delta_{a k}$, the right-hand side of the optimality condition for the stochastic PFE may takes different values among the paths between a specific OD pair. There is 
a possibility that the path flow pattern derived from the stochastic PFE is influenced more by the observed link flow constraint than by the logit-type assignment principle.

Thirdly, as described above, $\sum \mu_{1 a} \delta_{a k}$, the right-hand side of equation (9b) is associated with the observed link along path $k$. If no traffic detector is installed along path $k$, the right-hand side of (9b) must be zero and the stochastic PFE cannot estimate the path flow pattern that is satisfied with the optimality condition (9b). Accordingly, each path must include at least a link, into which a traffic detector is installed and operated.

\subsection{Determination of Parameter $\theta$}

The determination of parameter $\theta$ is similar to that of the SUE assignment. It may be interpreted as a measure of drivers' sensibility to the path costs; by varying this parameter it is possible to represent the different traveller behaviours on the network. The influence of the second part of the objective function (7) grows with the increase in the parameter. In the limit as $\theta \rightarrow \infty$, the user equilibrium flow comes out. This corresponds to a situation where travellers are extremely sensitive to the path cost, or closely say, the travellers have the perfect information about the actual travel cost. In reality, the value of the parameter $\theta$ is uniquely determined by the optimal flows, and may therefore be calibrated in a practical application, for instance from the observed flows. This paper takes the parameter as 0.1, like that in Bell and Iida (1997).

\section{Solution by Iterative Balancing}

Consider the Lagrange equation (8). The saddle point theorem says that at the optimum it is minimized with respect to the primal variables and maximized with respect to the dual variables. In the light of this theorem, the optimal solution may be searched by the sequentially optimizing the Lagrange function in terms of the dual and the primal. In other words, given initial values of $\boldsymbol{\mu}, \boldsymbol{\lambda}$, note so-called Lagrange problem.

$$
D\left(\boldsymbol{\mu}_{0}^{(i)}, \boldsymbol{\mu}_{1}^{(i)}\right)=\min L\left(\mathbf{h}^{(i)}, \boldsymbol{\mu}_{0}^{(i)}, \boldsymbol{\mu}_{1}^{(i)}\right)
$$

The solution of path flow $\mathbf{h}^{(i)}$ only relies on the temporal dual values of $i$-th iteration. The iterative procedure ended when the value of Lagrange function cannot further be improved. Otherwise, the new dual $\left(\boldsymbol{\mu}_{0}^{(i+1)}, \boldsymbol{\mu}_{1}^{(i+1)}\right.$ ) can be formulated, which may make Lagrange function ameliorable.

On the basis of this mind, the developed model of PFE is advantageous in its simplicity of calculation of flow and cost on relevant travel paths. By introducing the link performance function, the iterative balancing procedure proposed by Bell \& Iida (1997) is modified as follows:
Step 1 initialization

$$
\mathbf{x} \leftarrow \mathbf{0}, \mu_{0} \leftarrow \mathbf{0}, \mu_{1} \leftarrow \mathbf{0}
$$

Step 2 balancing cost

$\mathbf{c} \leftarrow \mathbf{c}(\mathbf{x})$

go to Step 4 if cost equilibrium

Step 3 balancing flow

Repeat

For all observed links $a$

$$
\begin{aligned}
& \ln (\mathbf{h}) \leftarrow \theta\left(\mathbf{A}_{1}^{\mathrm{T}} \boldsymbol{\mu}_{1}-\mathbf{C}-\mathbf{A}_{0}^{\mathrm{T}} \boldsymbol{\mu}_{0}\right) \\
& \mu_{1 a} \leftarrow \mu_{1 a}+\left[\ln v_{a}-\ln \left(\mathbf{A}_{1} \mathbf{h}\right)_{a}\right] / \theta
\end{aligned}
$$

For all unobserved links $a$

$$
\begin{aligned}
& \ln \mathbf{h} \leftarrow \theta\left(\mathbf{A}_{1}^{\mathrm{T}} \boldsymbol{\mu}_{1}-\mathbf{C}-\mathbf{A}_{0}^{\mathrm{T}} \boldsymbol{\mu}_{0}\right) \\
& \quad \text { if } s_{a}<\left(\mathbf{A}_{0} \mathbf{h}\right)_{a} \text { then } \\
& \quad \mu_{0 a} \leftarrow \mu_{0 a}+\left[\ln \left(\mathbf{A}_{0} \mathbf{h}\right)_{a}-\ln \left(s_{a}\right)\right] / s_{a} \\
& \text { delay occurring } \\
& d_{a} \leftarrow \mu_{a}
\end{aligned}
$$

until convergence

Step 4 output flow and delay

\section{Numerical Example}

\subsection{Single OD Case}

In order to confirm the basic characteristic of the PFE, the first example shows a very simplified situation in which only an OD pair is considered. The network is shown in Fig.4, which is also used in Bell and Iida (1997).

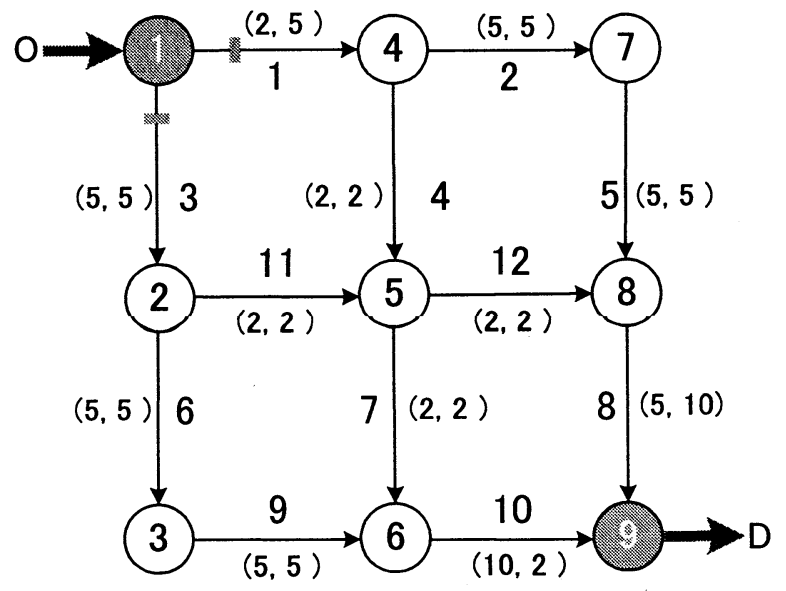

Fig. 4 Network of example 1

The free travel time and capacity are given in the brackets. There is only one OD pair between node 1 and node 9. It is assumed that traffic detectors are installed into links 1 and 3. Accordingly, every path from origin 1 to destination 9 includes an observed link. Since the detectors are installed in the upstream of each link, the congestion at the downstream of link is assumed to have no effect on the observed data. The observed link flows of link 1, 3 are assumed equal to their capacities. In this numerical example, the 
observed flows of both link 1 and link 3 are assumed to be 5 . The link performance function to estimate the link running time is, $t_{a}=t_{a 0}+0.01 x_{a}^{2}$, where, $t_{a 0}$ denotes the free travel time of the link $a$, and corresponds to the first item of the bracket illustrated in Fig. 4; $x_{a}$ represents the flow of link $a$. The parameter $\theta$ is assumed 0.1 .

Table 2. Estimated link flow and cost

\begin{tabular}{cccccc}
\hline link\# & capacity & flow & cost & time & delay \\
\hline $\mathbf{1}$ & 5 & 5.0 & 2.3 & 2.3 & 0 \\
2 & 5 & 3.0 & 5.1 & 5.1 & 0 \\
$\mathbf{3}$ & 5 & 5.0 & 5.8 & 5.75 & 0 \\
4 & 2 & 2.0 & 12.1 & 2.3 & 9.8 \\
5 & 5 & 3.0 & 5.1 & 5.1 & 0 \\
6 & 5 & 3.0 & 5.1 & 5.1 & 0 \\
7 & 2 & 2.0 & 12.1 & 2.3 & 9.8 \\
8 & 10 & 5.0 & 5.1 & 5.05 & 0 \\
9 & 5 & 3.0 & 5.1 & 5.1 & 0 \\
10 & 10 & 5.0 & 2.0 & 2.02 & 0 \\
11 & 2 & 2.0 & 9.2 & 2.29 & 6.9 \\
12 & 2 & 2.0 & 9.0 & 2.3 & 6.7 \\
\hline
\end{tabular}

Table 3. Estimated path cost and flow

\begin{tabular}{lccccc}
\hline \multicolumn{1}{c}{ path } & cost & time & delay & flow & Prob \\
\hline $1-4-7-10$ & 28.5 & 8.9 & 19.6 & 1.0 & 0.102 \\
$1-4-8-12$ & 28.5 & 11.9 & 16.5 & 1.0 & 0.102 \\
$1-2-5-8$ & 17.5 & 17.5 & 0 & 3.0 & 0.308 \\
$3-7-10-11$ & 29.0 & 12.4 & 16.7 & 1.0 & 0.098 \\
$3-8-11-12$ & 29.0 & 15.4 & 13.6 & 1.0 & 0.098 \\
$3-6-9-10$ & 18.0 & 18.0 & 0 & 3.0 & 0.292 \\
\hline
\end{tabular}

Table 2 shows the estimated link flows and the corresponding costs. As mentioned above, the link travel cost is composed of the running time and the overload delay. Because the observed flows of link 1 and 3 are equal to their capacities, it is assumed that the congestion does not occur on these links. The flowdependent PFE model forecasts the occurrence of congestion in the links $4,7,11$ and 12 due to the limited capacities of these links.

Table 3 shows the path cost including the delay, path flow and path choice probability. The path choice probability is calculated by using logit-type model expressed by equation (4), and the path travel time is inclusive of running time and delay on the path. The delays are found mostly in the paths including the links with the shorter running time under free flow condition but the lower capacities. A comparison between the path flow estimated and the path choice probability suggests that the flow pattern in this example almost follows the logit-type assignment principle. This suggestion is different from what we discuss in section 2.3. In this example, only an OD flow between node 1 and 9 is considered, and the flows of link 1 and 3, are observed by the detectors. No other traffic detector is installed into the downstream links of link 1 and 3. This is the reason why the stochastic PFE model gives the estimated path flows that follow the logit-type assignment principle.

\subsection{Multiple OD Case}

In the second example, the flow-dependent PFE model is applied to the network with the multiple OD pairs. Fig. 5 shows the network including four OD pairs: $(1,7),(1,9),(3,7)$, and $(3,9)$.

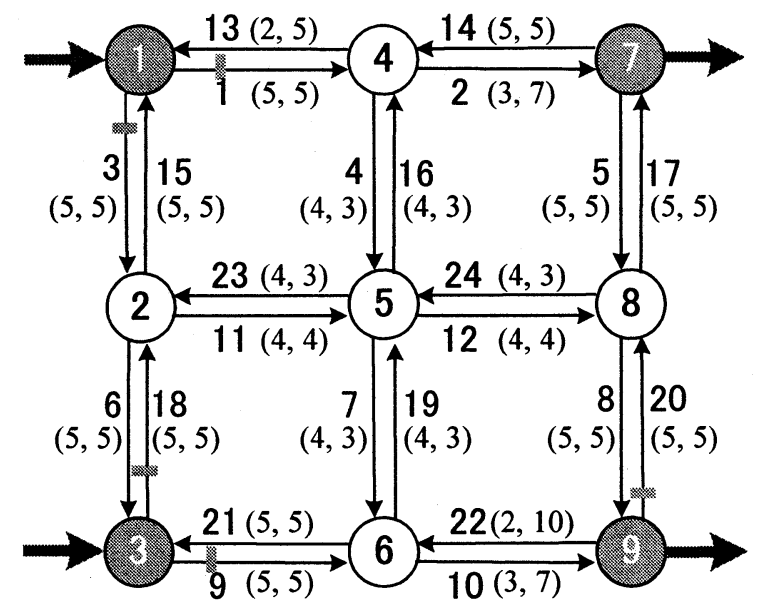

Fig. 5 Network of example 2

\begin{tabular}{|c|c|c|}
\hline link & Patten 1 & Pattern 2 \\
\hline 1 & 5 & 5 \\
\hline 3 & 5 & 5 \\
\hline 9 & 5 & 5 \\
\hline 18 & 5 & 5 \\
\hline 20 & $x$ & 2 \\
\hline
\end{tabular}

Two patterns of link flow observations are prepared for analyzing the influence of allocation of traffic detectors on the flows estimated by the PFE model. Table 4 shows the link flow observations. Here the standard BPR function is used to estimate the link running time. Table 5 shows the path flow and cost of OD pair $(1,9)$ estimated by using the PFE model. Also, Table 5 includes the logit choice probability estimated by equation (6). It can be found that the distribution of path flows from node 1 to 9 does not completely follow the path choice probability derived from the logit choice model that requires the more flow is assigned to the path of the less cost. In pattern 1 , for example, about three-fifth of the flows from node 1 to 9 gather in path 3-6-9-10, however the corresponding logit choice probability the path is less than two-fifth. As mentioned in section 2.3, there is a possibility that the path flow pattern derived from the stochastic PFE is influenced more by the observed link 
flow constraint than by the logit-type assignment principle. In other words, the estimated flows of the observed links must be equal to the flows measured by the traffic detectors. This is one of reasons why the path flow pattern obtained by using the stochastic PFE does not completely follow the logit-type assignment principle.

Table 5. Estimated flow, cost and delay of paths

\begin{tabular}{lccccccc}
\hline Paths in & \multicolumn{3}{c}{ Pattern 1 } & & \multicolumn{3}{c}{ Pattern 2 } \\
\cline { 2 - 4 } \cline { 6 - 8 } OD $(1,9)$ & Flow & Cost & Prob & & Flow & Cost & Prob \\
\hline $1-4-7-10$ & 0.36 & 17.01 & 0.260 & & 0.26 & 17.22 & 0.274 \\
$1-4-12-8$ & 0.28 & 19.37 & 0.205 & & 0.21 & 19.17 & 0.225 \\
$1-2-5-8$ & 0.29 & 18.97 & 0.213 & & 0.22 & 18.97 & 0.230 \\
$3-11-7-10$ & 0.56 & 29.95 & 0.071 & & 0.69 & 37.81 & 0.035 \\
$3-11-12-8$ & 0.44 & 32.31 & 0.056 & & 0.57 & 39.76 & 0.029 \\
$3-6-9-10$ & 2.99 & 19.88 & 0.195 & & 2.48 & 20.02 & 0.207 \\
\hline
\end{tabular}

Table 6. Estimated origin destination matrix

\begin{tabular}{ccccccc}
\hline & & \multicolumn{2}{c}{ Pattern 1 } & & \multicolumn{2}{c}{ Pattern 2 } \\
\cline { 2 - 3 } \cline { 6 - 7 } $\mathrm{O}$ & $\mathrm{D}$ & 9 & & 7 & 9 \\
\hline 1 & 2.08 & 4.93 & & 2.06 & 4.42 \\
3 & 4.93 & 2.07 & & 6.46 & 1.07 \\
\hline
\end{tabular}

In pattern 2 , it is assumed that the flow of link 20 is additionally observed. Table 5 suggests that the estimated path flow distribution of pattern 2 is different from that of pattern 1 . The PFE seems to be sensitive to the observed link flows because only one additional observation of link flow changes the path flow distribution drastically. Judging from the numerical example here, two contradictory opinions might be drawn. It could be said that the PFE can greatly capture the characteristic fluctuation of network flow; on the other hand, the PFE might deviate network flow state, if the observed link flows contain the large error. The resultant OD flows in two patterns are summarized in Table 6. It can be seen that the OD flows are also different between these two patterns. In the situation where an observed link flow is composed of the flows of various paths, the distributions of both path flows and OD flows estimated by using the stochastic PFE model are determined more by the pattern of the observed link flows than by the logit-type assignment principle.

\section{Consideration About Detector Allocation}

As generally known, the PFE processes available data collected from detectors, and provide the network information. Road users tend to concern about this kind of information to get their destination with shorter and/or reliable travel time, while transportation managers concern about the information to operate adaptive control policies, in order to maintain good quality of transportation service. The accuracy of estimated information is therefore exceptionally critical for both the road users and the road transportation managers. The success of the PFE is considered to depend on the quality of input data, the number and locations of traffic detectors. Section 2.3 concludes that every path under consideration should include at least an observed link into which the traffic detector is installed. Here we try to answer the question about the appropriate locations into which the traffic detectors should be installed, by applying the sensitivity analysis of SUE assignment, in order for the efficient use of the PFE.

There is a possibility that collected data is indifferent to network flow variations if traffic counters are not properly configured, so that output of the PFE might underestimate network flow. The sensitivity analysis is applied to supply some hint on the configuration of detectors from the viewpoint of influence of OD variation on link flow. We assume that the flow fluctuation at certain time slice is the variation in origin destination demand.

\subsection{Sensitivity for Stochastic User Equilibrium}

A Stochastic User Equilibrium assignment model will be applied to probe the importance of every link between a particular OD pair. So the same objective function in equation (7) is retentive, but the constraints are replaced by

$\mathbf{Q}=\mathbf{B h} \quad(\mathbf{u}) \quad$ (OD demand conservation)

$\mathbf{A h} \leq \mathbf{s} \quad(\mu) \quad$ (link capacity constraint)

where $\mathbf{Q}$ represents the OD demand matrix, B represents the incidence relationship of OD flow and path flows, $\mathbf{A}$ the incidence relationship of link and path flows, again, $\mathbf{A}=\left[\mathbf{A}_{0}, \mathbf{A}_{1}\right]^{\mathrm{T}} . \mathbf{u}, \boldsymbol{\mu}$ are the vectors of dual variables in expressions (12a) and (12b). At the optimum of the capacity-constrained SUE model governed by equations (7a) and (12), the differential relationship of input and output can be established as

$$
\begin{aligned}
& \delta \mathbf{C}=-\frac{1}{\theta} \nabla^{2} f(\mathbf{h}) \delta \mathbf{h}+\mathbf{B}^{\mathrm{T}} \delta \mathbf{u}-\mathbf{A}_{a}{ }^{\mathrm{T}} \delta \mathbf{\mu} \\
& \delta \mathbf{Q}=\mathbf{B} \delta \mathbf{h} \\
& \delta \mathbf{s}_{a}=\mathbf{A}_{a} \delta \mathbf{h}
\end{aligned}
$$

The first equation is the result of differentiating the optimality condition, the second is the differential equation of OD flow conservation and the third is the differential equation of the active capacity constraint. the subscript $a$ identifies the set of active link constraints. In the vector and the matrix notation, the variation in the demand/supply arguments, which are input data, are related to the variation in the primal and dual variables as follows:

$$
\left[\begin{array}{l}
\delta \mathbf{C} \\
\delta \mathbf{Q} \\
\delta \mathbf{s}_{a}
\end{array}\right]=\left[\begin{array}{ccc}
-\frac{1}{\theta} \nabla^{2} f & \mathbf{B}^{\mathrm{T}} & -\mathbf{A}_{a}^{\mathrm{T}} \\
\mathbf{B} & \mathbf{0} & \mathbf{0} \\
\mathbf{A}_{a} & \mathbf{0} & \mathbf{0}
\end{array}\right]\left[\begin{array}{l}
\delta \mathbf{h} \\
\delta \mathbf{u} \\
\delta \boldsymbol{\mu}
\end{array}\right]
$$

or in inverse matrix written as 


$$
\left[\begin{array}{l}
\delta \mathbf{h} \\
\delta \mathbf{u} \\
\delta \boldsymbol{\mu}
\end{array}\right]=\left[\begin{array}{lll}
\mathbf{J}_{11} & \mathbf{J}_{12} & \mathbf{J}_{13} \\
\mathbf{J}_{21} & \mathbf{J}_{22} & \mathbf{J}_{23} \\
\mathbf{J}_{31} & \mathbf{J}_{32} & \mathbf{J}_{33}
\end{array}\right]\left[\begin{array}{l}
\delta \mathbf{C} \\
\delta \mathbf{Q} \\
\delta \mathbf{s}_{a}
\end{array}\right]
$$

The last equation gives the sensitivities of path flow to OD demand and network performances. The variations in demand and supply have accordingly the following effect on flows along paths and links:

$\delta \mathbf{h}=\mathbf{J}_{11} \delta \mathbf{C}+\mathbf{J}_{12} \delta \mathbf{Q}+\mathbf{J}_{13} \delta \mathbf{s}_{a}$

$\delta \mathbf{x}=\left(\mathbf{A J}_{11}\right) \delta \mathbf{C}+\left(\mathbf{A J}_{12}\right) \delta \mathbf{Q}+\left(\mathbf{A J}_{13}\right) \delta \mathbf{s}_{a}$

where $\mathbf{x}$ indicates the link flow vector. The coefficients of three items in the RHS of equation (13b) indicate the sensitivity of link flows to the path travel time, the trip table and the link capacity. Here we have the particular interests in the middle one, $\mathbf{A} \mathbf{J}_{12}$, which also illustrates the importance order in links along the specific path. A successful detector allocation will actually capture link flow change in response to OD demand adjustment. Obviously the links, which hold the larger value among the elements of vector $\mathbf{A} \mathbf{J}_{12}$, becomes the candidate ones to set traffic counts, because detected data from such positions can greatly capture the characteristic attributes of network flow fluctuation.

\subsection{Test Example}

The same network shown in Fig. 4 is used to investigate the importance order of links for the singular OD pair, concerning the traffic detector allocation problem. The link flow sensitivity to the OD demand is estimated by using equation (13b) and the results are listed in Table 7.

Table 7. Link flow sensitivity to OD demand

\begin{tabular}{cccccccccccc}
\hline 1 & 2 & 3 & 4 & 5 & 6 & 7 & 8 & 9 & 10 & 11 & 12 \\
\hline 0.5 & 0.2 & 0.5 & 0.3 & 0.2 & 0.2 & 0.3 & 0.5 & 0.2 & 0.5 & 0.3 & 0.3 \\
\hline
\end{tabular}

It is revealed that links $1,3,8,10$ with sensitivity 0.5 , ought to be relatively important, compared with other links. Considering that there are six paths between the OD pair and that every path must have at least an observed link with the traffic detector, link 1 and 3, or 8 and 10, are suggested to have the traffic detector. So the allocation of counts in the first numerical example shown in section 4.1 can be regarded as one of feasible detector settings.

The speculation on the traffic detector allocation presented here is very simple, and it must be pointed out that such discussions are not sufficient for the practical application of the PFE. Note that influence coefficients in the sensitivity equation (13b) are associated with both network topography and decision behavior (shown in the objective function), which are worthy of further study. At least one detector being installed at every path under consideration is only one kind of the necessary conditions. These insights would be reported in the future studies.

\section{Conclusions}

This study proposes an improved stochastic PFE (Path Flow Estimator) that considers explicitly traffic congestion on the network. Basically, the PFE model can estimate the path flow and the OD flow by using the observed link flows. The link travel cost is assumed to consist of the flow-dependent running time and the overload delay. Due to the strong influence of the observed link flow constraint, the distribution of estimated path flows is determined more by the observed link flow than by the logit-type assignment principle adopted in the PFE. This characteristic of the stochastic PFE model has been confirmed through two cases of numerical examples. A modified iterative balancing procedure is set out the equivalent convex programming problem.

To overcome the sensitivity of the PFE to the observation error, an alternative propose is to allow the variation of the observation flow in certain intervals. Then, the observed link flow constraint requiring that the estimated link flows must coincide with the observed ones, should be replaced by the relaxed constraint that requires the estimated link flows must exist within the predetermined range of which the median is the observed link flow.

The further subjects will apply the improved PFE to the evaluating of the network performance measure and the connection of travel time reliability and network connectivity, and exploit the detector allocation problem for the practical use of the PFE.

\section{References}

1) 飯田恭敬，朝倉康夫 1988,2 レベル最適化から みた交通システム計画問題,ネットワークに関す る交通流理論および計画手法に関する体系的研究 成果報告書, pp. 67-70

2) Bell, M. G. H. and Iida, Y. (1997). Transportation Network Analysis, pp.114-148 John Wily \& Sons

3) Inoue, H. (1986). Traffic assignment on a network with congested flows, Proceedings of JSCE, No365/ IV-4, 125-133

4) Bell, M. G. H. et al. (1997). A stochastic user equilibrium path flow estimator, Transportation Research, 5C, 197-210

5) Sherali et al. (1994). A linear programming approach for synthesizing origin-destination trip tables from link traffic volumes, Transportation Research, 28B, pp.213-233

6) Yang, H. Sasaki, T. and Asakura, Y. (1992) Estimation of origin-destination matrices from link traffic counts on 


\title{
A Stochastic Flow-dependent Model for Path Flow Estimation
}

\author{
By Lin Cheng ${ }^{*}$ Yasunori Iida ${ }^{* *}$ Nobuhiro Uno ${ }^{* * *}$
}

This study proposes an improved stochastic PFE (Path Flow Estimator) that considers explicitly traffic congestion on the network. Basically, the PFE model can estimate both the path and the OD flows from the observed link flows. The link travel cost is assumed to consist of the flow-dependent running time and the overload delay. Due to the strong influence of the observed link flow constraint, the distribution of estimated path flows is determined more by the observed link flow than by the logit-type assignment principle adopted in the PFE. This characteristic of the stochastic PFE model has been confirmed through two cases of numerical examples. And then a modified iterative balancing procedure is set out the equivalent convex programming problem.

経路交通量推定のための確率的フローディペンデントモデル

程＼cjkstart琳・飯田＼cjkstart恭敬・宇野 伸宏

本研究では道路ネットワーク上の交通混雑を明示的に考慮可能とするため, 改良を施した経路交通量推 定手法について提案している. 基本的に，この方法では観測リンク交通量に基づき，経路交通量ならび にOD交通量を推定可能である。リンクの旅行費用は，その值が交通量に依存する走行時間ならびに需 要の集中に起因寸る遅れから構成されると仮定している．観測リンク交通量制約の影響が強いため，推 定経路交通量の分布は，ロジット型配分原則よりむしろ観測リンク交通量の影響を強く受けて決定され る.この手法が有する上記の特徵については，2 種類の数值計算例を通して確認された．また，等価な 凸計画問題を解くため, 改良型の反復調整法が適用された. 